\title{
Phase Locked Harmonic Generation in the Opaque Region of GaAs
}

\author{
C. Cojocaru ${ }^{1}$, L. R. Suñé ${ }^{1}$ M. Scalora ${ }^{2}$, and J. Trull ${ }^{1}$

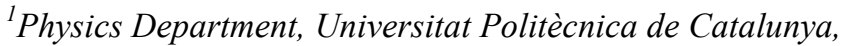 \\ Rambla Sant Nebridi 22, Terrassa (Barcelona), Spain \\ ${ }^{2}$ Charles M. Bowden Research Centre, AMRDEC, RDECOM, Redstone Arsenal, AL 35898-5000, USA \\ Tel: , Fax:, e-mail: crina.maria.cojocaru@gmail.com
}

\begin{abstract}
We demonstrate second and third harmonic generation from a GaAs substrate, well-below the absorption edge, in both transmission and reflection geometries. The pump is tuned at $1064 \mathrm{~nm}$, in the transparency range, while the $\mathrm{SH}$ and the TH signals are tuned in the opaque spectral range of GaAs, at $532 \mathrm{~nm}$ and $355 \mathrm{~nm}$, respectively. As expected, we find that the polarization of the generated signals is sensitive to the polarization of the pump. In our experiment, we work far from the phase matching condition and we account for both surface and bulk contributions, and show that the surface-generated SH components can be more intense than bulk-generated SH signals. The experimental results are contrasted with numerical simulations that include these two factors, using a hydrodynamic model that accounts for all salient aspects of the dynamics, including surface and bulk generated harmonic components.
\end{abstract}

Keywords: nonlinear optics, harmonic generation, phase locked second harmonic.

\section{INTRODUCTION}

The use of semiconductors in the process of fabrication of actual nano-devices such as $\mathrm{GaAs}, \mathrm{GaP}$ or $\mathrm{Si}$ is at the front edge of nowadays technology, exploiting the properties of light at nanometric scale in new and surprising ways. Light localization at dimensions smaller than the wavelength is possible using the properties of electromagnetic field enhancement by plasmonic resonances in metal-dielectric boundaries or in metamaterials or under conditions of epsilon near zero. At these scales the usual theory describing the nonlinear (NL) effects of electromagnetic fields should be revisited and analyzed.

The NL response of these semiconductors has been traditionally studied under phase-matching conditions, where the energy conversion from the fundamental field (FF) beam to its harmonics reach its maximum efficiency. Under such conditions, the leading NL polarization term corresponds to the bulk contribution described through the quadratic susceptibility tensors. However, other contributions to the NL polarization arise from electric quadrupole or magnetic dipole interactions and must be considered when the bulk term is drastically reduced, as happens in very thin dielectric layers, where the phase-mismatch loose its sense. It is well known that under phase mismatch conditions, when a pump pulse crosses an interface between a linear and a NL medium, there are always three generated second harmonic (SH) components: one is generated backward (reflection) into the linear medium and the other two components are generated forward (transmission). This phenomenon was discussed first in [1], where it has been shown that the general solution of the Maxwell's equations with NL polarization sources has a homogeneous and an inhomogeneous solution. This solution shows two contributions of the transmitted SH field. The homogeneous component travels with the expected group velocity given by material dispersion at the SH wavelength: $k(2 \omega)=k_{0}(2 \omega) n(2 \omega)$, where $k_{0}(2 \omega)$ is the wavenumber for the $\mathrm{SH}$ in vacuum. The inhomogeneous component is captured by the pump pulse and experiences its same effective dispersion as the FF: $2 k_{0}(\omega) n(\omega)$, where $k_{0}(\omega)$ is the wavenumber of the FF in vacuum. The inhomogeneous component, named phase locked (PL), was experimentally reported for the first time in 1990 [2] and theoretically studied in details in 2007 [3].

A more intriguing situation arises for the case when the FF beam is tuned in the transparency range of the material, but the SH frequency lye well below the absorption edge. Since the inhomogeneous SH solution "sees" the refractive index of the FF wave, the imaginary part of the refractive index corresponds also to that of the FF. This leads to the counter-intuitive result that the PL harmonic components can propagate in the opaque region of the material. The existence of this propagation was experimentally demonstrated in [4]. One year later it has been shown that the PL SH component generated in opaque materials can be amplified in a cavity resonant only for the FF wavelength [5]. However, as far as we know, the contribution of different NL source terms on the harmonic PL components has been never studied.

In this paper we go beyond the previous studies and we study more deeply this process in order to infer which are the different mechanisms leading to the PL SH generation. We demonstrate that a measurement of the polarization and angular dependence of the generated SH signal can give detailed information of the relative contribution of each one of these mechanisms. Experimental and theoretical simulations are contrasted, using a hydrodynamic model that accounts for all salient aspects of the dynamics, including surface and bulk generated harmonic components. 


\section{MODEL AND NUMERICAL SIMULATIONS}

Detailed numerical simulations of these phenomena has been developed by Scalora et al. solving Maxwell's equations with the NL polarization term, using a Fast Fourier Transform beam propagating method, described in [3]. Although this model was able to describe the PL harmonic generation and propagation in opaque materials, the model used to describe the nonlinearity only includes the bulk contribution to the NL polarization, usually described by the second and third order electric susceptibility as:

$$
P(t)=\varepsilon_{0}\left[\chi^{(1)} E(t)+\chi^{(2)} E^{2}(t)+\chi^{(3)} E^{3}(t)+\ldots\right]
$$

where $\chi^{(1)}$ is the linear susceptibility, $\chi^{(2)}$ and $\chi^{(3)}$ are the second- and third-order NL susceptibilities and $\varepsilon_{0}$ is the permittivity of free space.

Besides this NL contribution arising from NL restoring forces, SH and TH fields can also be generated at the surface of the medium as a result of breaking of translational symmetry. This phenomenon can be understood following the theoretical approach outlined in [6]. In general, NL sources may be specified in terms of a complex dielectric function described by a combined Drude-Lorentz, which contains a mix of free and bound electrons having one or more resonances. For typical GaAs substrates, free carrier doping is very low $\left(10^{14} \mathrm{~cm}^{-3}\right.$ to $\left.10^{17} \mathrm{~cm}^{-3}\right)$, so that the Drude portion may be neglected. Therefore, in our case only bound electrons are assumed to play a role in the surface SH generation. Neglecting for the moment the NL restoring forces $\left(\chi^{(2)}\right)$, Newton's second law for one species of bound electrons leads to the following effective polarization equation for bound charges:

$$
\ddot{\boldsymbol{P}}_{\boldsymbol{b}}+\gamma_{b} \dot{\boldsymbol{P}}_{\boldsymbol{b}}+\omega_{0, b}^{2} \boldsymbol{P}_{\boldsymbol{b}}=\frac{n_{0, b} e^{3}}{m_{b}^{*}} \boldsymbol{E}+\frac{e}{m_{b}^{*} c} \dot{\boldsymbol{P}}_{\boldsymbol{b}} \times \boldsymbol{H}
$$

where $\mathbf{P}_{\mathrm{b}}$ is the polarization, $\dot{\mathbf{P}}_{\mathrm{b}}$ is the bound current density, $\mathbf{H}$ is the magnetic field, e is the electron charge, $m_{\mathrm{b}}^{*}$ is the bound electron's effective mass and $c$ is the speed of light. When we develop Eq. (2), we found the following expressions for the $\mathrm{SH}$ polarization envelope function in the undepleted pump approximation:

$$
\ddot{\boldsymbol{P}}_{b, 2 \omega}+\tilde{\gamma}_{b} \dot{P}_{b, 2 \omega}+\widetilde{\omega}_{0, b}^{2} \boldsymbol{P}_{b, 2 \omega} \approx \frac{n_{0, b} e^{2} \lambda_{0}^{2}}{m_{b}^{*} c^{2}} \boldsymbol{E}_{b, 2 \omega}+\frac{e \lambda_{0}}{m_{b}^{*} c^{2}}\left(\boldsymbol{E}_{\omega} \boldsymbol{\nabla} \boldsymbol{P}_{b, \omega}+\left(\dot{\boldsymbol{P}}_{b, \omega}-i \omega \boldsymbol{P}_{b, \omega}\right) \times \boldsymbol{H}_{b}\right)
$$

where $\lambda_{0}=1 \mu \mathrm{m}$ and some coefficients have been scaled:

$$
\tilde{\gamma}_{b, N \omega}=\frac{\lambda_{0}}{c}\left(\gamma_{b}-N i \omega\right), \quad \widetilde{\omega}_{0, b, N \omega}^{2}=\frac{\lambda_{0}}{c}\left(\omega_{0}^{2}-(N \omega)^{2}+i \gamma_{b} N \omega\right)
$$

where $N=2$ is an integer that denotes the $\mathrm{SH}$.

Eq. (3) suggests that in the absence of bulk nonlinearities, we still have SHG coming from the gradient of the polarization, which is non-zero at the surface and from the magnetic field (Lorentz force), which has both surface and bulk components. NL restoring forces may be also introduced and the total second order NL polarization, described by the model presented above may be written in a simplified manner as follows:

$$
\boldsymbol{P}_{N L}(2 \omega) \approx \chi^{(2)} \boldsymbol{E}^{2}+\frac{e \lambda_{0}}{m_{b}^{*} c^{2}}\left(\boldsymbol{E}_{\omega} \boldsymbol{\nabla} \boldsymbol{P}_{b, \omega}+\left(\dot{\boldsymbol{P}}_{b, \omega}-i \omega \boldsymbol{P}_{b, \omega}\right) \times \boldsymbol{H}_{b}\right)
$$

where $\chi^{(2)}$ is a tensor quantity. Eq. (5) shows three main contributions that are expected to participate in the resulting NL signal: (i) the NL bulk contribution arising from the anharmonic potentials (first term); (ii) a purely surface contribution coming from the divergence of the polarization (second term), and (iii) the NL contribution from the magnetic part of Lorentz's force (third term).

To infer the relative contribution of each one of these terms, we introduce the NL polarization in the Maxwell's equations and we use the theoretical model described in $[6,7]$. We consider here the propagation of a $50 \mathrm{fs}$ fundamental pulse tuned at $1064 \mathrm{~nm}$ in a $10 \mu \mathrm{m}$ wafer of GaAs, oriented with the (001) crystallographic direction perpendicular to the surface. We generate SH signal at $532 \mathrm{~nm}$. The real and imaginary parts of the permittivity of GaAs are shown in Fig. 1b. The GaAs is transparent for wavelengths above $900 \mathrm{~nm}$ and strongly absorptive below it, which means that the FF is pumped in the transparency range (at $1064 \mathrm{~nm}$ ), while the SH $(532 \mathrm{~nm})$ is tuned well below the absorption edge of GaAs. Through the simulation model we are able to modify the input parameters such as angle of incidence, wavelength and polarization, pulse width and intensity and the thickness of the GaAs substrate. The adjusting parameters are the NL coefficients and the effective mass of the bound electrons. The polarization of the incident FF beam is selected to be either TE: $\left(\mathrm{E}_{\mathrm{x}}, 0,0\right)$ or TM: $\left(0, \mathrm{E}_{\mathrm{y}}, \mathrm{E}_{\mathrm{z}}\right)$, being $\mathrm{z}$ the propagation direction (see Fig. 1c) and the incident intensity $2 \mathrm{GW} / \mathrm{cm}^{2}$.

We have performed several simulations, varying the input beam polarization and angle of incidence. Figures 2a-2c show the transmitted (red) simulated PL SH efficiencies as a function of the angle of incidence. In Fig. 2a the polarization of the FF field is set at TM and we represent only the TM component of the SH. The transmission efficiency is of order $10^{-7}$ and is maximum at $\sim 60^{\circ}$. In Fig. $2 \mathrm{~b}$ the polarization of the FF is still TM, but now we plot only the TE component of the SH. TE-polarized SH signal is generated by the bulk $\chi^{2}$ of GaAs. This transmission efficiency is two orders of magnitude smaller compared to the case illustrated in Fig. 2a and the maximum is now found around $50^{\circ}$. The TE fundamental generating TM-SH is illustrated in Fig. $2 \mathrm{c}$ and it refers to a surface-generated, TM-polarized SH signal. Transmission efficiency is of order $10^{-8}$ and the curve 
shows a peak at $80^{\circ}$. For TE(FF)-TE(SH) configuration, no $\mathrm{SH}$ is generated.

The first important aspect of these results is the appearance of a transmitted SH despite being propagated in the opaque region of the material, with conversion efficiencies of the order of $10^{-7}$. The second important aspect is that we can see different active polarization channels.

\section{EXPERIMENTAL RESULTS}

In the next step, the relative contribution of the different NL sources in the mechanism of generation and propagation of harmonic radiation in opaque region of GaAs was experimentally demonstrated. The schematic representation of our setup is shown in Fig. 1a. The fundamental beam is generated by a fiber laser emitting pulses of $13 \mathrm{ps}$ at $1064 \mathrm{~nm}$. The typical intensities used in the experiments were of the order of $1.6 \mathrm{GW} / \mathrm{cm}^{2}$. A half-wave plate, placed at the laser output, controls the polarization of the fundamental beam, selecting TE or TM polarization. The analysis of the generated SH polarization is performed by a second polarizer, placed after the sample. The sample, an undoped GaAs wafer of $500 \mu \mathrm{m}$ thick cut with the (001) direction perpendicular to the surface is set in a rotary support that precisely controls the incident angle. Figure 1c shows the definition of TM and TE polarizations with respect to the incident plane and to the crystallographic axes of the GaAs. The SH at $532 \mathrm{~nm}$ is generated at the entrance in the GaAs slab and, since it falls in the opaque region of the material, only the PL component can propagate and be finally transmitted through the sample.

The most critical part of this experiment is the detection of the $\mathrm{SH}$, with efficiencies of the order of $10^{-7}-10^{-9}$. In order to detect the faint SH signal a photomultiplier was used together with a spectral filter with a $20 \mathrm{~nm}$ band pass transmission band around the SH frequency. To avoid SHG from other surfaces in the setup different filters to attenuate the fundamental beam were placed just after the GaAs wafer. To separate the very weak harmonic radiation from the stronger fundamental beam a prism was used after the GaAs wafer and before the photomultiplier. A stop was placed after the prism to block the fundamental radiation. A detailed calibration procedure was performed in order to give a quantitative estimation of the efficiencies of the process. The described setup allows the measurement of efficiencies of the order of $10^{-9}$.

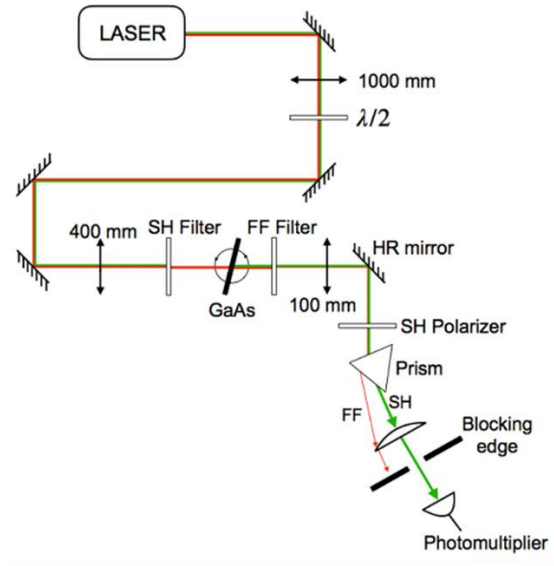

(a)

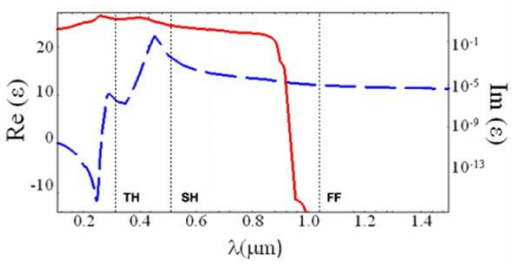

(b)

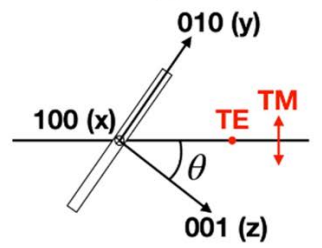

(c)

Figure 1: (a) Experimental setup for measuring the transmitted SH signals; (b) Real (blue) and imaginary (red) parts of the permittivity of GaAs; (c) Definition of TM and TE polarizations with respect to the incident plane and the crystallographic axes of GaAs.

Figures $2 \mathrm{a}-2 \mathrm{~d}$ show the experimental results (blue) and simulations (red) of the transmitted SH efficiency as a function of the incident angle, for the following polarization combinations: TM(FF)-TM(SH) - Fig. 2a, $\mathrm{TM}(\mathrm{FF})-\mathrm{TE}(\mathrm{SH})$ - Fig. $2 \mathrm{~b}$ and TE(FF)-TM(SH) - Fig. 2c. For TE-polarized FF and SH no signal was observed for any angle of incidence, which is in accordance with the theoretical simulations. As can be immediately inferred, the component of SH generated and propagated through a $500 \mu \mathrm{m}$ thick sample of material in the presence of strong absorption corresponds to the propagation of the PL component. The comparison experiment - simulation shows a good fit. The presence of a TM component of the SH is a clear indication of the nonnegligible contribution of the surface and Lorentz's terms in this process. In our measurements we obtain efficiencies of the order of $10^{-7}$ and $10^{-9}$. For what the bulk contribution is concerned (TM(FF)-TE(SH)), the transmitted SH efficiency is proportional to $10^{-9}$, which tells us that the surface and Lorenz terms play a more important role in the generation of SH than the bulk for our GaAs sample. The adjusting parameters of the model, namely the quadratic susceptibility and the effective mass were kept the same in all simulations. From the results we can see that the angular dependence is in good correspondence with the numerical model for all cases. The comparison between the relative magnitude between the different mechanisms obtained in the experiment is shown in Fig. $2 d$. 


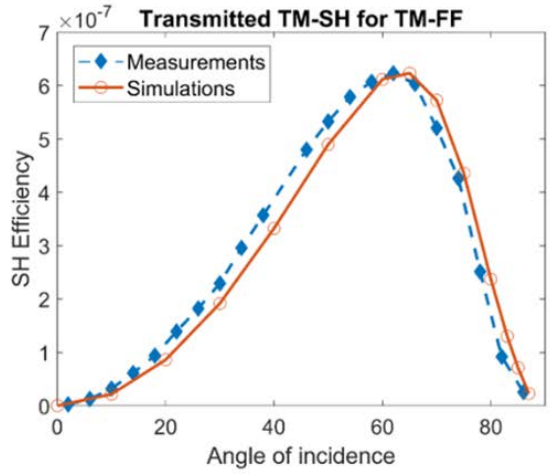

a) TM-polarized FF and TM-polarized SH.

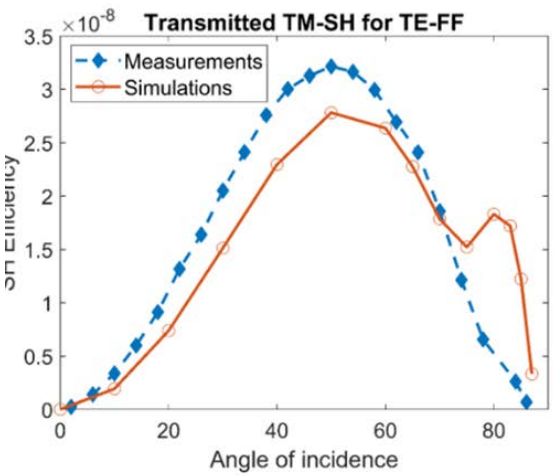

c) TE-polarized FF and TM-polarized SH.

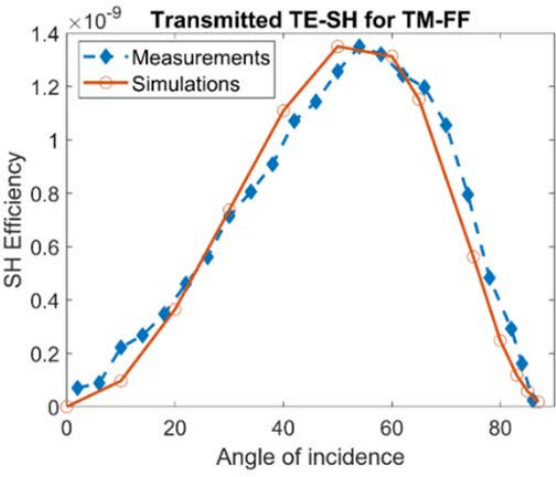

b) TM-polarized FF and TE-polarized SH.

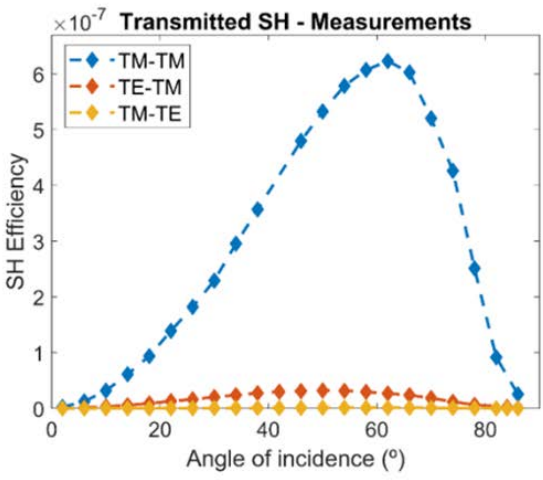

d) Transmission curves.

Figure 2: Calculated (red) and measured (blue) SH transmission efficiencies as a function of the angle of incidence.

\section{CONCLUSIONS}

Phase locked component of the SH generated signal and its propagation in the opaque region of GaAs has been experimentally measured, with efficiencies of order $10^{-7}-10^{-9}$. This study went beyond previous ones and shows different mechanisms leading to the PL harmonic generation: different components in transmission, corresponding to both surface and bulk contributions are measured and contrasted. The surface-generated SH components is, in this case, more intense than bulk-generated SH signal. The experimental data fits well theoretical predictions despite resonances appearing in the measured curves which have been justified. This fact gives us information about the sample as the fitting has been done by adjusting the second-order susceptibilities and the effective mass of bound electrons.

\section{REFERENCES}

[1] J. A. Armstrong, N. Bloembergen, J. Ducuing, and P. S. Pershan, "Interactions between light waves in a nonlinear dielectric," Phys. Rev. 127, 1918 (1962).

[2] L. D. Noordam, H. J. Bakker, M. P. de Boer, H. B. van Linden van den Heuvell, "Second-harmonic generation of femtosecond pulses: Observation of phase-mismatch effects," Opt. Lett. 15, 24 (1990).

[3] V. Roppo, M. Centini, C. Sibilia, M. Bertolotti, D. de Ceglia, M. Scalora, N. Akozbek, M. J. Bloemer, J. W. Haus, O. G. Kosareva, and V. P. Kandidov, Phys. Rev. A 76, $033829,2007$.

[4] M. Centini, V. Roppo, E. Fazio, F. Pettazzi, C. Sibilia, J. W. Haus, J. V. Foreman, N. Akozbek, M. J. Bloemer, and M. Scalora, "Inhibition of linear absorption in opaque materials using phase-locked harmonic generation," Phys. Rev. Lett. 101, 113905 (2008).

[5] V. Roppo, C. Cojocaru, F. Raineri, G. D’Aguanno, J. Trull Y. Halioua, R. Raj, I. Sagnes, R. Vilaseca, and M. Scalora, "Field localization and enhancement of phase-locked second- and third-order harmonic generation in absorbing semiconductor cavities," Phys. Rev. A 80, 043834 (2009).

[6] M. Scalora, M. A. Vincenti, D. de Ceglia, V. Roppo, M. Centini, N. Akozbek, and M. J. Bloemer, "Secondand third-harmonic generation in metal-based structures," Phys. Rev. A 82, 043828 (2010).

[7] M. Scalora, M. Vincenti, D. de Ceglia, N. Akozbek, V. Roppo, M. Bloemer, and J. W. Haus, "Dynamical model of harmonic generation in centrosymmetric semiconductors at visible and UV frequencies," Phys. Rev. A 85, 053809 (2012). 